JSCER

ISSN: 2708-1079

\title{
Effective Reengineering Computing Model For Ethiopian Health Care Center
}

\author{
Dr.Rajasekar Sundaram \\ Department Of Information Technology, TVET College, Ethiopia.
}

\begin{tabular}{lll}
\multicolumn{3}{l}{ Article Information } \\
Received & 16 March 2020 \\
Revised & $:$ & 21 March 2020 \\
Accepted & $:$ & 25 March 2020 \\
Published & 10 April 2020
\end{tabular}

Corresponding Author:

Dr.Rajasekar Sundaram

Email: jhsudhamuru@gmail.com

\begin{abstract}
The ordinary information get to control framework is to keep up the specific sharing composite Personal Health Information Records (PHIRs)in health center, operations from different health centers in cloud major research looks into in the present IT. A PHIR administration license a patient to making, overseeing, and controlling the individual wellbeing information in one spot through the web, which has made the extra room, recovery, and appropriation of the clinical data progressively effective. Uncommonly, every patient is secure the full control of clinical records and can impart the wellbeing information to an enormous scope of clients with social insurance donors and relatives. Because of the significant expense of building and keep up secured and specific server, numerous PHIR administrations are redistributed to make accessible by client from specialist organizations. Decentralized server farms the emission of $\mathrm{CO} 2$ is high and nature get spoiled and polluted by making reusable datacenters the data's or information's can shared through the PHIR's. By this we can keep away from the high emission of $\mathrm{CO}$, the adaptability; accessibility and similarity are increments according to Moore's law. To share the cutting edge data's from other server farm information with reusable asset and e-Health Care Service in Ethiopian health center.
\end{abstract}

Keywords: Big Data, Data Center, Green computing, Heath Care.

Copyright $\odot$ 2020: Dr.Rajasekar Sundaram. This is an open access distribution, and reproduction in any medium, provided Access article distributed under the Creative Commons Attribution License the original work is properly cited License, which permits unrestricted use.

Citation: Dr.Rajasekar Sundaram. "Effective Reengineering Computing Model for Ethiopian Health Care Center, "Journal of Science, Computing and Engineering Research,1(1), 21-24, Mar-Apr 2020.

\section{INTRODUCTION}

As of late, individual Personal Health Information Record (PHIR) has risen as a patient-driven model of wellbeing data trade. A PHIR administration permits a patient to make, oversee, and control her own wellbeing information in one spot through the web, which has made the capacity, recovery, and sharing of the clinical data progressively effective. Particularly, every patient is guaranteed the full control of her clinical records and can impart her wellbeing information to a wide scope of clients, including human services suppliers, relatives or companions. Because of the significant expense of building and keeping up specific server farms, numerous PHIR administrations are re-appropriated to or gave by outsider specialist organizations.

The principle concern is about whether the patients could really control the sharing of their touchy individual Personal Health Information Record (PHIR), particularly when they are put away on an outsider server which individuals may not completely trust [2]. Then again, despite the fact that there exist social insurance guidelines, for example, HIPAA which is as of late corrected to fuse business partners, cloud suppliers are normally not secured substances. Then again, because of the high estimation of the touchy delicate individual Personal Health Information Record (PHIR) the outsider stockpiling servers are frequently the objectives of different malevolent practices which may prompt introduction of the PHIR [3]

The objective of patient-driven protection is regularly in conflict with versatility in a PHIR framework. The approved clients may either need to get to the PHIR for individual use or expert purposes. Instances of the previous are relative and companions, while the last can be clinical specialists, drug specialists, and analysts, and so forth. We allude to the two classes of clients as close to home and expert clients, individually. The last has possibly enormous scope; should every proprietor herself be legitimately answerable for dealing with all the expert clients, she will handily be overpowered by the key administration overhead. Moreover, since those clients' entrance demands are commonly flighty. There has been a significant increment in the normal temperature of the earth in the previous century. This ascent in temperature is ascribed with the impacts of a dangerous atmospheric deviation achieved by the amassing of ozone depleting substances (GHG) in the air. The explanation behind expanded GHG, essentially Carbon Dioxide (CO2), is a direct result of the expanded vitality utilization which brings about outflow of contaminations. Common disasters 


\title{
Effective Reengineering Computing Model For Ethiopian Health Care Center
}

\author{
Available at https://jscer.org
}

like tropical storms, floods and changes in the ocean levels are credited to the $\mathrm{CO} 2$ fuelled nursery impact. It is assessed that during the most recent 30 years the $\mathrm{CO} 2$ outflows have gone up by $73 \%$. Ethiopia is positioned fifth among the nations in the rundown of worldwide GHG outflow, with USA and China contributing around multiple times emanation than that of Ethiopia [4]. The Kyoto Protocol of 1997, which was marked by more than 160 nations, including Ethiopia, approaches all nations to diminish their discharges of nursery gasses by 5\%, from the 1990 level, continuously 2012. Numerous administrations around the globe, including Ethiopia have found a way to lessen vitality utilization and discharges. Ethiopia is resolved to lessen carbon power by 20-25\% among 2005 and 2020. As of now, the ICT part all around represents 0.9 metric gigatons of $0047 \mathrm{HG}$ emanations every year, or about $2 \%$ of all out worldwide discharges; which incorporates PCs, servers, cooling hardware, fixed and portable communication, neighborhood (LAN) and printers.

Table I Global CO2 emissions

\begin{tabular}{|c|c|c|}
\hline Overview & $\begin{array}{l}\text { Emission of } \\
\text { Gigatons Gas CO2e } \\
2012\end{array}$ & $\begin{array}{l}\text { Emission of Gigatons } \\
\text { CO2e } 2020\end{array}$ \\
\hline $\begin{array}{l}\text { Global CO2 } \\
\text { emissions }\end{array}$ & 41.2 & 51.9 \\
\hline $\begin{array}{l}\text { Total ICT } \\
\text { footprint }\end{array}$ & 0.62 & 1.43 \\
\hline $\begin{array}{l}\% \text { of Global } \\
\text { emissions }\end{array}$ & $1.70 \%$ & $2.80 \%$ \\
\hline
\end{tabular}

II. RELATED WORKS

Social insurance IT area spending in Ethiopia is presently limited by client populace; for instance, in excess of 60 percent of spending on servers and programming. In any case, numerous non-renewable energy source and material assets are situated in rustic territories, which have less than 20 percent of figuring clients and records for under $25 \%$ of IT spending in Ethiopia. Working up Ethiopia's National distributed computing framework throughout the following a very long while. As a result of ongoing advances in the social insurance foundation and system figuring, moving the servers and datacenters to rustic could be beneficial. There's not by any means a logical response to the topic of how much vitality an including vitality utilized by the customer gadget, organize, and datacenter [11].

Making an away from of information access between various clients dependent on the idea of the clients and qualities or relationship with the information proprietor is the end idea of yield for this framework. Right now, normal isolation of clients will be done and they didn't discuss the appropriation of information among numerous databases. On the off chance that the information is excessively perplexing, this framework won't clarify how the relativity of information is kept up between the tables and they didn't discuss the exhibition recovery of huge information. Special approval nature given to the information proprietor which a genuine imperfection and it ought not be case [14]. The explanation is, the information ought to be given to give to a portion of the legislature related individuals to give increasingly tie down nature to running the administration foundations. If there should be an occurrence of Emergency, the patients face will be identified through Fractal Segmentation idea to recover the pertinent information of him and thusly a programmed SMS will be sent to the gatekeeper demonstrating the client's information is gotten to by the crisis group [21]

Working up Ethiopia's national distributed computing framework throughout the following quite a few years. Due to ongoing advances in the human services framework and system registering, moving the servers and datacenters to rustic could be invaluable. There's not by any means a logical response to the topic of how much vitality an including vitality utilized by the customer gadget, arrange and datacenter.

Server farm's today normally offer force the board and cooling highlights including fans that accelerate or back off varying, just as increasingly proficient force supplies. Sharp edge servers are more productive than ordinary rack servers since cutting edges share a solitary frame, just as force and cooling [17]. IT is a noteworthy client of power, and is liable for over $2 \%$ of the world's carbon impression vigorously on IT, for example, banks and in numerous other office businesses, IT is regularly liable for well over portion of all power utilization for little and medium estimated malls. Express Shopping pamphlet advises the clients with late advancements, and can give accommodation to shopping. However the bulletin forces risk to asset and condition, for its shading ink printing paper [5].

\section{PROPOSED ARCHITECTURE}

Developing Ethiopian E-card database which eventually contains details of every Ethiopian citizen, an individual identity is defined in terms of human attributes is name, gender, age and address. But human data alone cannot guarantee uniqueness. Uniqueness is likely by linking human attributes with bio-metric characteristics like thumbprint and iris patterns of the single, so no need to create novel users data's. The real time working of Ethiopian E-card. Following is the Operating model of Ethiopian E-card and real time flow of how the transactions take place in Ethiopian E-card model. With current development in technology, it is now likely to make a digital uniqueness for an individual in a huge population using bio- metric characteristics (thumbprint and iris) which can be confirmed online. Each uniqueness can be assigned numerous identity tokens of numerous kinds which can be used suitably for authentication as her the business need of service rendered. 


\title{
Effective Reengineering Computing Model For Ethiopian Health Care Center
}

\author{
Available at https://jscer.org
}

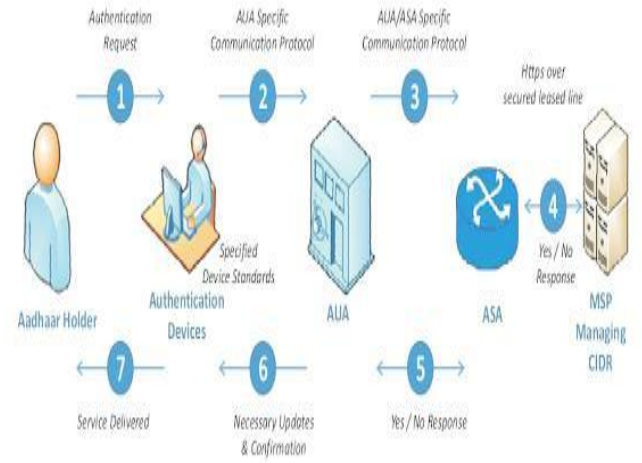

Figure 1General Architecture of Health Information System

\section{A. System Architecture}

The main objective of proposed framework is to deliver secure patient-centric PHIR access and effective key management at the similar time. The key idea is to distribute the system into many security areas (namely, public sectors (PUSs) and personal sectors (PSSs)) according to the different users' data access requirements. The PUSs consist of customers who make access based on their expert roles, such as doctors, nurses and medical researchers. PUS can be drawn to an autonomous sector in the society, such as the health care, management or insurance sector. For each PSS, its users are individually connected with a data owner (such as household members or close friends), and they make admissions to PHIRs based on access rights assigned by the owner.

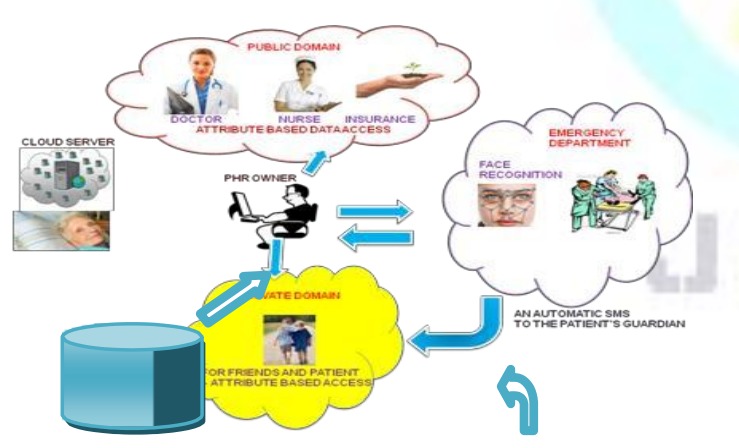

Figure 2 Proposed Prototype Architecture

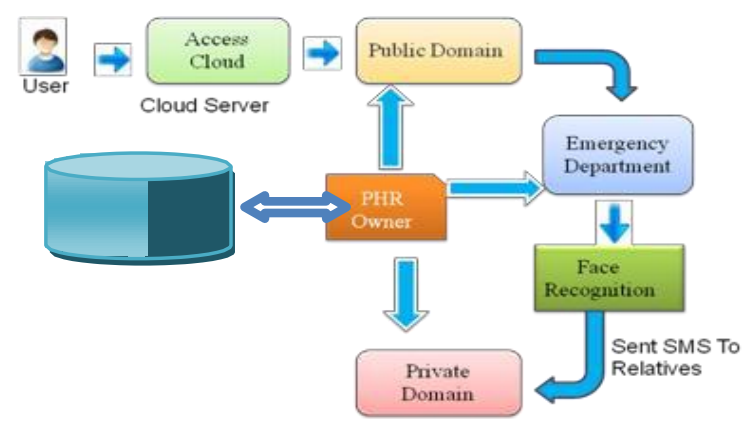

Figure 3 Green Computing System Architecture
This research endeavor to study the patient-centric, secure sharing of PHIRs stored on semi-trusted servers, and focus on addressing the complicated and challenging key management issues. To address the key management challenges, we conceptually divide the users in the system into two types of domains, namely public and personal sectors. In particular, the majority professional users are managed distributive by attribute authorities in the former, while each owner only needs to manage the keys of a small number of users in her personal domain. In this way, our framework can simultaneously handle different types of PHIR sharing applications' requirements, while incurring minimal key management overhead for both owners and users in the system. In addition, the framework enforces write access control, handles dynamic policy updates, and provides break-glass access to PHIRs under emergence scenarios.

This research demonstrate how our frame-work works using a concrete example. Suppose PHIR owner Alice is a patient associated with hospital A. After she creates a PHIR file F1 (labelled as "PHIR; medical history; allergy; emergency"), she first encrypts it according to both F1's data labels, and a role-based file access policy. This policy can be decided based on recommended settings by the system, or Alice's own preference. It look like p1 $:=$ "“(profession=physician $) \wedge($ specialty=internalmedicine $) \wedge($ or ganization=hospital A)". She also sends the break-glass key to the ED. In addition, Alice determines the access rights of users in her PSS, which can be done either on-line or off-line. For example, she may approve her friend Bob's request to access files with labels \{personal info or $\{$ medical history\}. Her client application will distribute a secret key with the access structure (personal info $\mathrm{V}$ medical history) to Bob. When Bob wants to access another file F2 with labels "PHIR - medical history medications", he is able to decryptF2 due to the "medical history" attribute. For another user Charlie who is a physician specializing in internal medicine in hospital B in the PUS, he obtains his secret key from multiple AAs. But he cannot decryptF1, because his role attributes do not satisfyP1.

Finally, an emergency room staff, Dorothy who temporarily obtains the break-glass key from ED, can gain access toF1 due to the emergency attribute in that key. The separation of PSS/PUS and data/role attributes reflects the real-world situation. First, in the PSS, a patient usually only gives personal access of his/her sensitive PHIR to selected users, such as family members and close friends, rather than all the friends in the social network. Different PSS users can be assigned different access privileges based on their relationships with the owner. In this way, patients can exert fine-control over the access for each user in their PSSs. Second, by our multi-domain and multi-authority framework, each public user only needs to contact AAs in its own PUS who collaboratively generates a secret key for the user, which reduces the workload per AA.

Page 23 


\title{
Effective Reengineering Computing Model For Ethiopian Health Care Center
}

\author{
Available at https://jscer.org
}

\section{CONCLUSION}

This highlights one of the key issues in Green IT responsibility. Green IT is such a large topic that it extends far beyond the data center or the IT department. It also affects, and is the responsibility of, end users and lines of business within the organization, the procurement function, and middle and senior management. Sustainability, in all its aspects, is a key business driver in the 21 st century. There is an increased realization across society that business practices and individual behavior needs to change. IT has a major role to play. As a major contributor to global carbon emissions its needs to get its own house in order, but more importantly the IT function needs to be more significantly involved in enabling the transition to a true low carbon economy. A novel framework of secure sharing of personal health records in cloud computing is proposed. Considering partially trustworthy cloud servers, we argue that to fully realize the patient-centric concept, patients shall have complete control of their own privacy through encrypting their PHIR files to allow fine-grained access. The framework addresses the unique challenges brought by multiple PHIR owners and users, in that we greatly reduce the complexity of key management while enhance the privacy guarantees compared with previous works. This research utilize ABE to encrypt the PHIR data, so that patients can allow access not only by personal users, but also various users from public sectors with different professional roles, qualifications and affiliations

\section{REFERENCES}

[1] Boldyreva, V. Goyal, and V. Kumar, "Identity-based encryption with efficient revocation," inACM CCS, ser. CCS '08, 2008, pp.417-426.

[2] Dong, G. Russello, and N. Dulay, "Shared and searchable encrypted data for untrusted servers," in Journal of Computer Security, 2010

[3] H. L" ohr, A.-R. Sadeghi, and M. Winandy, "Securing the e-health cloud," inProceedings of the 1st ACM International Health Informatics Symposium, ser. IHI '10, 2010, pp. 220-229

[4] J. Hur and D. K. Noh, "Attribute-based access control with effi-cient revocation in data outsourcing systems, "IEEE Transactions on Parallel and Distributed Systems, vol. 99, no. PrePrints, 2010.

[5] L. Ibraimi, M. Petkovic, S. Nikova, P. Hartel, and W. Jonker, "Ciphertext-policy attribute-based threshold decryption with flexible delegation and revocation of user attributes," 2009.

[6] X. Liang, R. Lu, and X. Lin, "Ciphertext Policy Attribute Based Encryption with Efficient Revocation," Technical Report BBCR, Univ. of Waterloo, 2011.

[7]J. Hur and D.K. Noh, "Attribute-Based Access Control with Efficient Revocation in Data Outsourcing Systems," IEEE Trans. Parallel and Distributed Systems, vol. 22, no. 7, pp. 1214-1221, July 2011.

[8] G. Zhao, C. Rong, J. Li, F. Zhang, and Y. Tang, "Trusted Data Sharing over Untrusted Cloud Storage Providers," Proc. IEEE Second Int'l Conf. Cloud Computing Technology and Science (CLOUDCOM'10), pp. 97-103, 2010.

[9]P.K. Tysowski and M.A. Hasan, "Towards Secure Communication for Highly Scalable Mobile Applications in Cloud
Computing Systems," Technical Report 33, Centre for Applied Cryptographic Research (CACR), Univ. of Waterloo, 2011.

[10] G. Ateniese, K. Fu, M. Green, and S. Hohenberger, "Improved Proxy Re-Encryption Schemes with Applications to Secure Distributed Storage," ACM Trans. Information and System Security, vol. 9, pp. 1-30, Feb. 2006.

[11] S. Jahid, P. Mittal, and N. Borisov, "EASiER: EncryptionBased Access Control in Social Networks with Efficient Revocation," Proc. Sixth ACM Symp. Information, Computer and Comm. Security (ASIACCS '11), pp. 411-415, 2011.

[12] Q. Liu, G. Wang, and J. Wu, "Clock-Based Proxy ReEncryption Scheme in Unreliable Clouds," Proc.41st Int'l Conf. Parallel Processing Workshops (ICPPW), pp. 304-305, Sept. 2012.

[13] J.-M. Do, Y.-J. Song, and N. Park, "Attribute Based Proxy Re- Encryption for Data Confidentiality in Cloud Computing Environments," Proc. First ACIS/JNU Int'l Conf. Computers, Networks, Systems and Industrial Eng. (CNSI), pp. 248-251, May 2011.

[14] S. Yu, C. Wang, K. Ren, and W. Lou, "Attribute Based Data Sharing with Attribute Revocation," Proc. Fifth ACM Symp. Information, Computer and Comm. Security (ASIACCS '10), pp. 261-270, 2010.

[15] Y. Ming, L. Fan, H. Jing-Li, and W. Zhao-Li, “An Efficient Attribute Based Encryption Scheme with Revocation for Outsourced Data Sharing Control," Proc. First Int'l Conf. Instrumentation, Measurement, Computer, Comm. and Control, pp. 516-520, 2011.

[16] S. Yu, C. Wang, K. Ren, and W. Lou, "Achieving Secure, Scalable, and Fine-Grained Data Access Control in Cloud Computing,” Proc. IEEE INFOCOM'10, pp. 534-542, 2010.

[17] K. Yang and X. Jia, "Attributed-Based Access Control for Multi- Authority Systems in Cloud Storage," Proc. IEEE 32nd Int'l Conf. Distributed Computing Systems (ICDCS), pp. 536-545, 2012.

[18] K. Yang, X. Jia, K. Ran, and B. Zhang, "DAC- MACS: Effective Data Access Control for Multi- Authority Cloud Storage Systems," Proc. IEEE INFOCOM, pp. 2895-2903, 2013.

[19] J. Wang, "Java Realization for Cipertext-Policy AttributeBased Encryption," http://github.com/wakemecn, 2012.

[20] G. Wang, Q. Liu, and J. Wu, "Hierarchical Attribute- Based Encryption for Fine-Grained Access Control in Cloud Storage Services," Proc. 17th ACM Conf. Computer and Comm. Security (CCS '10), pp. 735-737, 2010.

[21] H. Rasheed, "Auditing for Standards Compliance in the Cloud: Challenges and Directions" The International Arab Journal of Information Technology, July 2003. 